\title{
Deficiency of liver sinusoidal scavenger receptors stabilin-1 and -2 in mice causes glomerulofibrotic nephropathy via impaired hepatic clearance of noxious blood factors
}

\author{
Kai Schledzewski, ${ }^{1}$ Cyrill Géraud, ${ }^{1}$ Bernd Arnold, ${ }^{2}$ Shijun Wang, ${ }^{3}$ \\ Hermann-Josef Gröne, ${ }^{3}$ Tibor Kempf, ${ }^{4}$ Kai C. Wollert, ${ }^{4}$ Beate K. Straub, ${ }^{5}$ \\ Peter Schirmacher, ${ }^{5}$ Alexandra Demory, ${ }^{1}$ Hiltrud Schönhaber, ${ }^{1}$ Alexei Gratchev, ${ }^{1}$ \\ Lisa Dietz, ${ }^{1}$ Hermann-Josef Thierse, ${ }^{1}$ Julia Kzhyshkowska, ${ }^{1}$ and Sergij Goerdt ${ }^{1}$
}

1Department of Dermatology, Venereology, and Allergology, University Medical Center and Medical Faculty Mannheim, University of Heidelberg, and Center of Excellence in Dermatology, Mannheim, Germany. ${ }^{2}$ Department of Molecular Immunology and ${ }^{3}$ Department of Cellular and Molecular Pathology, German Cancer Research Center (DKFZ), Heidelberg, Germany. ${ }^{4}$ Department of Cardiology and Angiology, Hannover Medical School, Hannover, Germany. ${ }^{5}$ Department of Pathology, University Clinic Heidelberg, University of Heidelberg, Heidelberg, Germany.

\begin{abstract}
Tissue homeostasis and remodeling are processes that involve high turnover of biological macromolecules. Many of the waste molecules that are by-products or degradation intermediates of biological macromolecule turnover enter the circulation and are subsequently cleared by liver sinusoidal endothelial cells (LSEC). Besides the mannose receptor, stabilin-1 and stabilin-2 are the major scavenger receptors expressed by LSEC. To more clearly elucidate the functions of stabilin-1 and -2 , we have generated mice lacking stabilin-1, stabilin-2, or both stabilin-1 and -2 (Stab1-/-Stab2-/- mice). Mice lacking either stabilin-1 or stabilin-2 were phenotypically normal; however, Stab1 ${ }^{-/}$Stab2 $2^{-/}$mice exhibited premature mortality and developed severe glomerular fibrosis, while the liver showed only mild perisinusoidal fibrosis without dysfunction. Upon kidney transplantation into WT mice, progression of glomerular fibrosis was halted, indicating the presence of profibrotic factors in the circulation of Stab1 $1^{-/ S t a b 2^{-/}}$mice. While plasma levels of known profibrotic cytokines were unaltered, clearance of the TGF- $\beta$ family member growth differentiation factor 15 (GDF-15) was markedly impaired in Stab1 $1^{-/}$Stab2 $2^{-/}$mice but not in either Stab1 $1^{-/-}$or Stab2 $2^{-/}$mice, indicating that it is a common ligand of both stabilin-1 and stabilin-2. These data lead us to conclude that stabilin-1 and -2 together guarantee proper hepatic clearance of potentially noxious agents in the blood and maintain tissue homeostasis not only in the liver but also distant organs.
\end{abstract}

\section{Introduction}

Tissue homeostasis and remodeling are accompanied by high turnover of all categories of biological macromolecules including proteins, proteoglycans, and polysaccharides as well as nucleic acids and lipids (1). During these processes, waste molecules are generated either as by-products of synthesis or as intermediates of degradation that accumulate locally and may spill over into the circulation. For the major constituents of the extracellular matrix, such as collagens or glycosaminoglycans, the degradation products may amount to several grams generated daily in the human organism, indicating the need for efficient removal and reusage. Similar considerations apply to aged plasma proteins such as coagulation factors or immunoglobulins. Surprisingly, nothing is known about the clearance of regulatory molecules such as hormones, growth factors, and other cytokines that circulate in the blood under physiological or pathological conditions such as pregnancy, inflammation, wound healing, or tumor growth. The first-line local degradation system for waste molecules comprises resident or immigrant tissue macrophages and the regional lymph

Authorship note: Kai Schledzewski and Cyrill Géraud contributed equally to this work. Sergij Goerdt and Julia Kzhyshkowska share senior authorship. Conflict of interest: The authors have declared that no conflict of interest exists. Citation for this article: J Clin Invest. 2011;121(2):703-714. doi:10.1172/JCI44740 nodes. Waste molecules that have entered the circulation are preferentially cleared by a specialized scavenger endothelial cell system, i.e., liver sinusoidal endothelial cells (LSEC), that has been highly conserved during evolution (2).

To fulfill their clearance functions, LSECs express 3 major types of scavenger receptors: (a) the macrophage mannose receptor binding denatured collagen (3) and collagen peptides preventing gelatin formation in the circulation, tissue plasminogen activator regulating fibrinolytic activity, and lysosomal enzymes that are recruited for further use in LSEC (4); (b) the Fcy receptors FcyRII in the human system (5) and FcyRIIB2 in the rat $(6,7)$; and (c) the major LSEC hyaluronan (HA) scavenger receptor. Upon identification and cloning of the stabilin family of fasciclin-like HA receptor homologs by our group (8), the identity of the LSEC HA scavenger receptor was established as stabilin-2 on the basis of HA affinity purification and sequencing (9). Stabilin-1 was first identified as MS-1 high-molecular weight antigen expressed by sinusoidal endothelial cells in human liver, spleen, and lymph nodes as well as by resident tissue macrophages $(10,11)$. Stabilin- 1 and stabilin-2 are multidomain type 1 transmembrane receptors that exhibit an identity of $55 \%$ on the protein level but show no DNA homology; the homology between man and mouse is $86 \%$ for stabilin- 1 and $79 \%$ for stabilin-2 and documents the high evolutionary conservation of these 2 proteins. Both stabilin- 1 and stabilin- 2 com- 
A WT

WT stab-1

stab-2

stab-dko
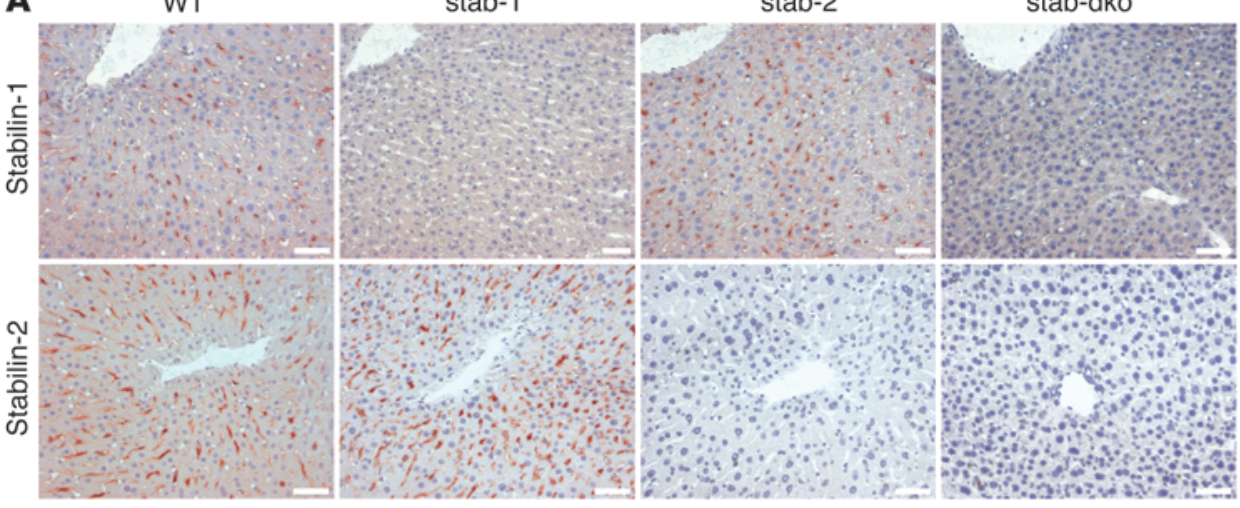

B

C57BL/6 survival

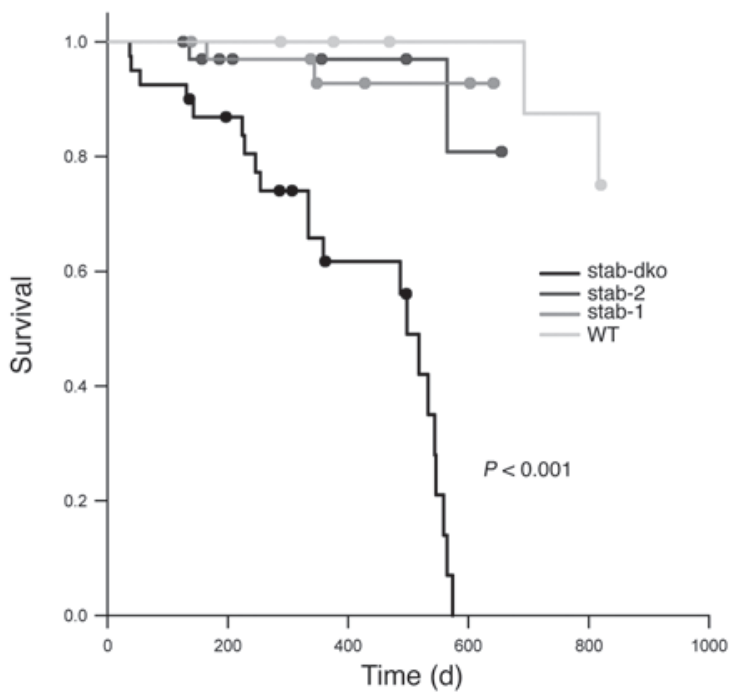

C

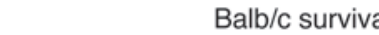

Figure 1

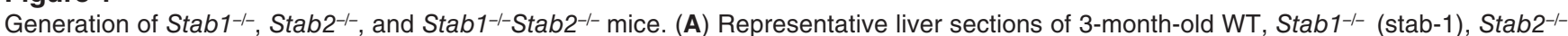
(stab-2), and Stab1-/-Stab2-/- (stab-dko) mice of C57BL/6 genetic background were stained by immunohistochemistry with antibodies against stabilin-1 (upper row) and stabilin-2 (lower row). WT sections show sinusoidal distribution of both stabilins. No stabilin-1 was detected in Stab1-1-,

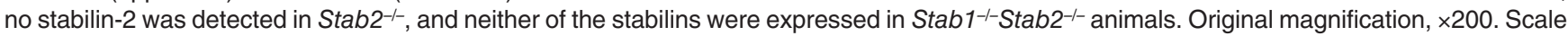

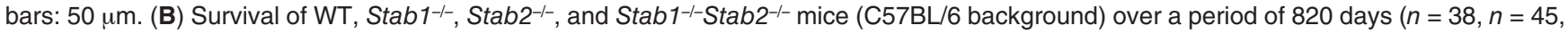

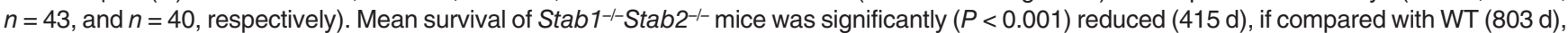
Stab1-/- $(615 \mathrm{~d})$, and Stab2 ${ }^{--}(625 \mathrm{~d})$ strains. Single-deficient mice displayed a tendency toward reduced lifespan $\left(\right.$ Stab $1^{-/-}[P=0.13]$ and

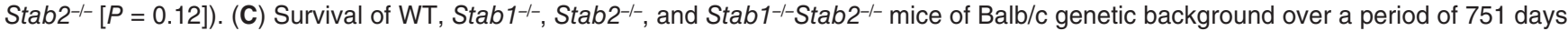
( $n=31, n=44, n=30$, and $n=102$, respectively). Mean survival of Stab1-/-Stab2-/- mice was dramatically reduced (269 d), if compared with WT (739 d), Stab1-/- $(584 \mathrm{~d})$, and Stab2-/- $(618 \mathrm{~d})$. Single-deficient mice displayed a tendency toward a reduced lifespan $($ Stab1-/- $[P=0.28])$ and $\left.S_{\text {Stab2 }}{ }^{--}[P=0.06]\right)$ that did not reach the level of confidence $(P<0.05)$. (B and $\left.\mathbf{C}\right)$ The survival data were analyzed by log-rank statistics, and pair-wise multiple comparison procedures were done by the Holm-Sidak method.

prise clusters of EGF-like (cell/matrix interactions) and fasciclin domains (protein/protein interactions) and $1 \mathrm{X}$-link domain (HA binding) in their extracellular parts.

While stabilin-2 avidly binds HA, stabilin-1 does not bind HA due to a nonfunctional X-link domain (12). Despite high structural similarity, the spectrum of ligands differs considerably between stabilin- 1 and stabilin-2. In addition to binding HA, stabilin- 2 binds and mediates endocytosis of AGE-modified proteins and collagen $\mathrm{N}$-terminal propeptides (12). At present, it is a controversial issue whether stabilin-2 also binds and mediates internalization of heparin $(13,14)$. In contrast, stabilin-1 binds and mediates endocytosis of acLDL, the angiogenic extracellular protein SPARC, and the hor- mone placental lactogen (PL), thereby regulating the extracellular concentration levels of these molecules (15-17). For internalization of their ligands in LSEC, stabilin-1 and - 2 use the constitutive clathrin-mediated endocytic pathway (12); clathrin-coated vesicles and stabilin- $1 / 2^{+}$early endosomes travel along microtubules organized in a very special net-like way linking the highly specialized morphology of LSEC to their endocytic function (18). In addition to its role in endocytosis, stabilin-1 acts as an intracellular cargo carrier (19-21) and is involved in leukocyte trafficking (22).

In order to further elucidate the functions of stabilin-1 and -2 in health and disease, we generated stabilin-1-deficient (Stab1-/-)

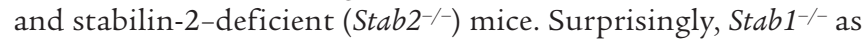


A

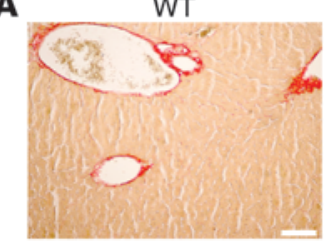

stab-2

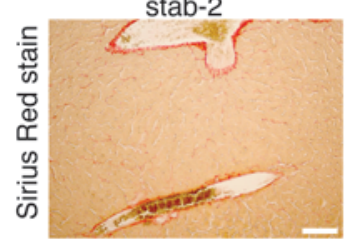

C Liver transaminases

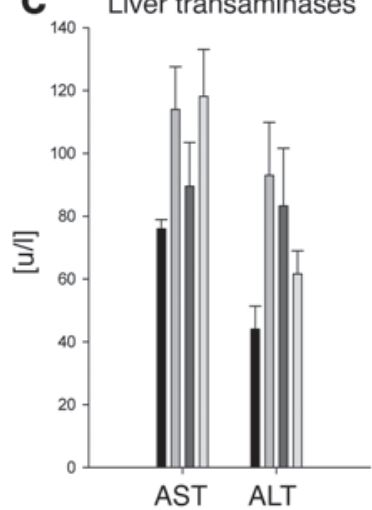

stab-1

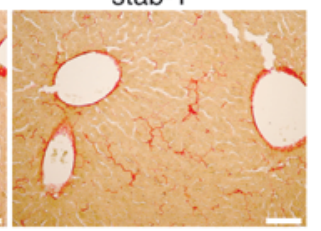

stab-dko

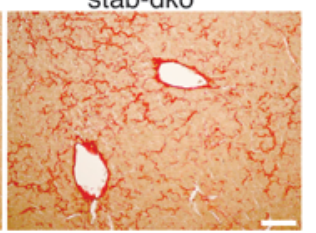

D
B

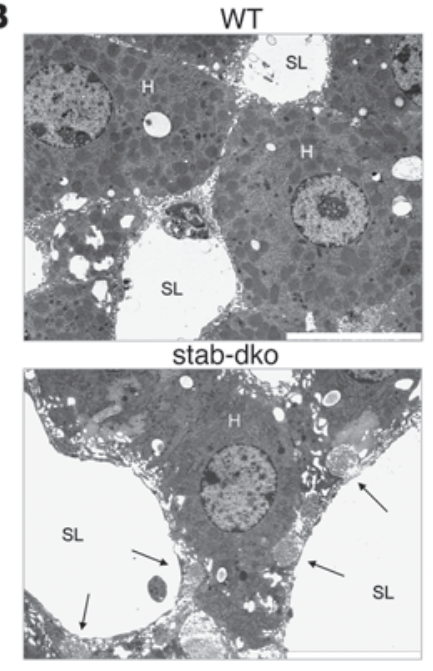

\section{Results}

Premature mortality of Stab1-/Stab2-/- mice. Mice homozygous

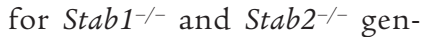
erated on C57BL/6 as well as $\mathrm{Balb} / \mathrm{c}$ genomic background (Supplemental Figures 1 and 2; supplemental material available online with this article; doi:10.1172/JCI44740DS1; for further details, see Methods) displayed no obvious physical or behavioral abnormalities and bred comparably to WT control animals. To determine whether the homologous multidomain proteins stabilin-1 and stabilin-2 exert important redundant functions, Stab1 $1^{-/}$Stab2 $2^{-/-}$ mouse strains were generated on both Balb/c and C57BL/6 genomic backgrounds by crossing single-deficient mice. The Stab1/-Stab2-/- genotype was confirmed regarding genomic DNA, as well as concerning mRNA and protein expression. Immunohistochemistry of liver specimens demonstrated also

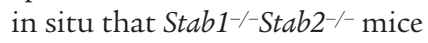
display a double deficiency for stabilin-1 as well as for stabilin-2 proteins in the liver sinusoids (Figure 1A); expression of stabilin- 1 or of stabilin- 2 in the liver sinusoids was not influenced by deficiency of stabilin-2 or stabilin-1, respectively. In order to test the cellular integrity of LSECs within the liver sinusoids of the various $\mathrm{KO}$ mice, LSEC marker proteins such as FcyRIIB2/CD32b (7) and LYVE-1 (23) were analyzed by well as Stab2-/- single-deficient mice did not show an obvious phenotype. As we could not exclude that redundant functions and common ligands of both stabilin- 1 and stabilin- 2 had hitherto eluded identification, we generated Stab1 $1^{-/}$Stab2 $2^{-/-}$mice. Indeed, Stab1 $1^{-/-}$Stab2 $2^{-/-}$mice exhibited premature mortality and developed severe glomerular fibrosis and albuminuria accompanied by mild perisinusoidal hepatic fibrosis. Kidney transplantation experiments further confirmed the notion that glomerulofibrotic nephropathy was due to one or several circulating factor(s) present in Stab1 $1^{-/}$Stab2 $2^{-/-}$mice. While HA serum levels were highly elevated in Stab2 $2^{-/}$single-deficient mice as well as

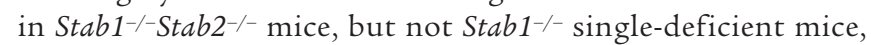
growth differentiation factor 15 (GDF-15), a distant TGF- $\beta$ family member, was identified here as a common endocytic ligand of both stabilin-1 and stabilin-2 whose clearance was substantially

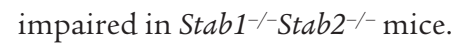

immunohistochemistry as well as immunofluorescent analysis and confocal microscopy. None of the independent LSEC markers displayed gross differences when Stab1 $1^{-1-S t a b 2^{-/-}}$mice were compared with Stab1 $1^{-/}$or Stab2-/- or WT control animals (data not shown).

In contrast to the single-deficient strains, both Balb/c Stab1-/-

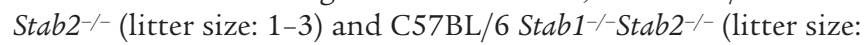
2-3) did not reach offspring rates of WT control animals (litter size: 8-10). Normal breeding rates were obtained, however, by

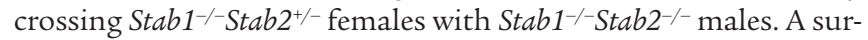

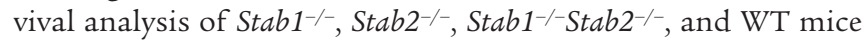
was performed over a time period of 700 days. Both Balb/c and C57BL/6 Stab1 $1^{-/-}$Stab2-/- strains suffered from reduced overall survival rates with a mean survival time of 415 days for C57BL/6

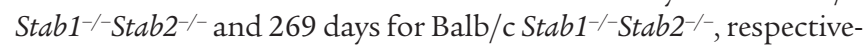
ly (Figure 1, B and C). The differences in overall survival rates were highly statistically significant when Stab1-/-Stab2-/- and single- 


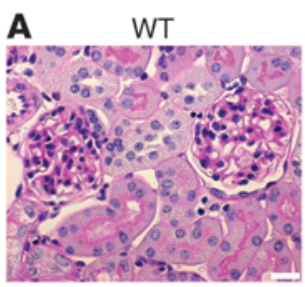

stab-2

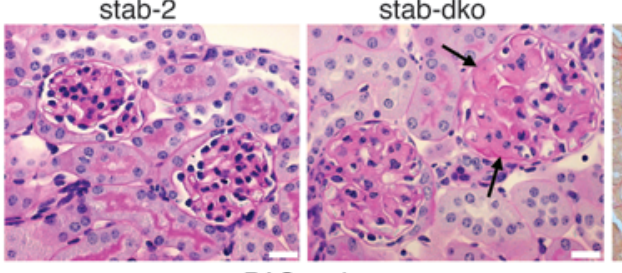

PAS stain

stab-1

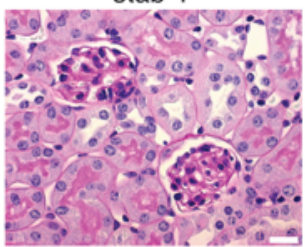

stab-dko
Albumine (urine)

D

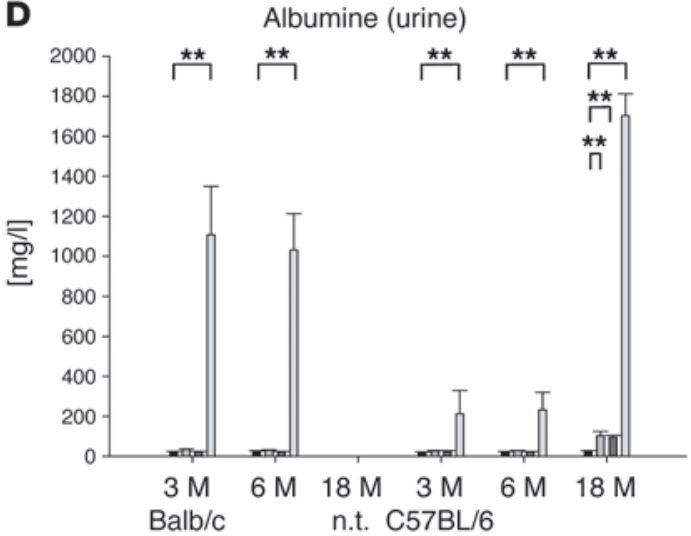

B

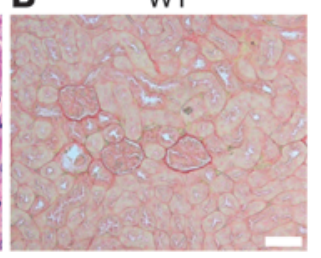

stab-dko

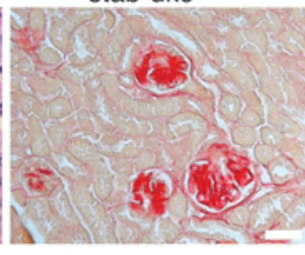

Sirius red stain

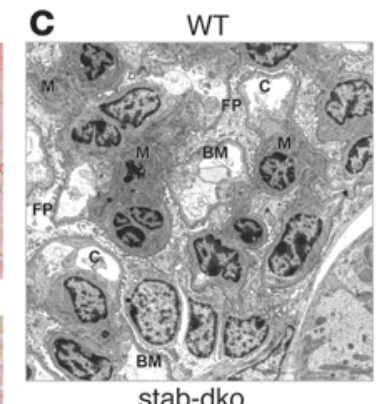
stab-dko

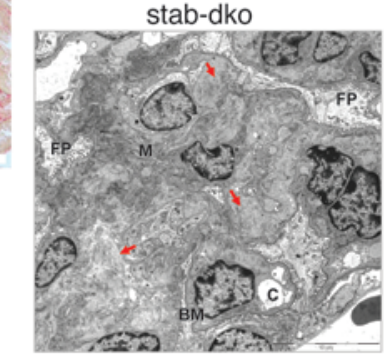

\section{Figure 3}

Kidney phenotype of stabilin-deficient mice. (A) Representative PAS-stained kidney sections from 3-month-old WT, Stab1-/-, Stab2-/-, and

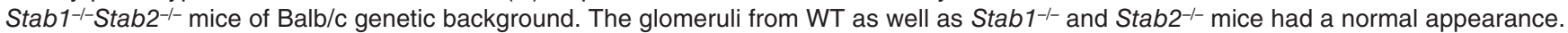

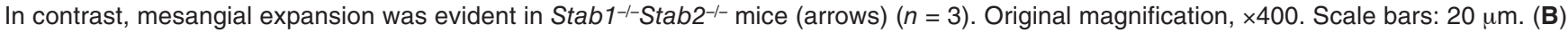
Representative kidney sections of 3-month-old WT and Stab1-1-Stab2 $2^{-1-}$ mice of C57BL/6 background were stained with Sirius red to identify the degree of fibrosis $(n=3)$. Strong Sirius red-positive staining indicating collagen deposition was detected only in Stab1 $1^{-/-S t a b 2^{-/-}}$glomeruli. The extent of the glomerulosclerosis was similar in Stab1--Stab2-/- mice of both Balb/c and C57BL/6 genetic background (not shown). Original magnification, $\times 200$. Scale bars: $50 \mu \mathrm{m}$. (C) Representative transmission EM images of 4-week-old Stab1-/-Stab2 ${ }^{-/}$glomeruli of Balb/c genetic background as compared with WT showed large deposits of fibrillar material (red arrows) and a severely enlarged mesangium (M) with narrowing of capillary lumens (C), partial loss of foot processes (FP) and regular basement membrane (BM). $n=3$. Original magnification, $\times 10,000$. Scale bars: $10 \mu \mathrm{m}$. (D) Urine of 3- and 6-month-old Balb/c and 3-, 6-, and 18-month-old C57BL/6 mice of all genotypes was collected and analyzed for


single-deficient animals. Data represent mean \pm SEM from 5 mice. ${ }^{* *} P<0.01$ relative to WT values.

deficient mice as well as WT controls were compared; in contrast, when Stab1 $1^{-/}$and Stab2 $2^{-/-}$mice were compared with WT control animals, no statistically significant differences in survival rates were found irrespective of the genetic background (C57BL/6 or $\mathrm{Balb} / \mathrm{c}$ ) (detailed analysis summarized in Supplemental Table 1). $\mathrm{Balb} / \mathrm{c}$ Stab1 $1^{-/-}$Stab2 $2^{-/}$mice sacrificed at an age of approximately the mean survival time (269 days) had developed incipient to overt dilated cardiomyopathy with or without heart failure and pulmonary congestion that might be the cause of premature mortality, while C57BL/6 Stab1-/-Stab2-/- mice sacrificed at an age of approximately the mean survival time (415 days) did not show signs of cardiac disease, indicating genetic variability between inbred strains.

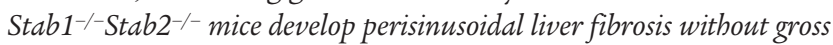
liver dysfunction. Although no gross abnormalities in liver structure or weight were noticed at first glance in any of the mouse strains, a detailed histological analysis of liver specimens was performed including H\&E, Sirius red, and silver staining. Whereas no differences were detected by H\&E and silver staining (not shown), Sirius red staining demonstrated a considerable increase in perisinusoi-

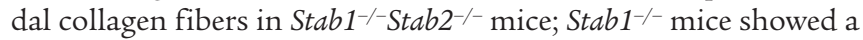
minor increase in Sirius red-positive fibers, while Stab2 $2^{-/-}$mice did not differ from control animals (Figure 2A). Sirius red-positive collagen fibers were first observed in 10-week-old Stab1 $1^{-/}$Stab2 $2^{-/}$mice and increased in number with age independent of the genetic background (C57BL/6 or Balb/c). Electron microscopy confirmed the development of large collagen fibers located directly beneath the abluminal side of the sinusoidal endothelial cell layer (Figure 2B).

To analyze whether the perisinusoidal liver fibrosis observed in Stab1 $1^{-1-S t a b 2^{-/-}}$mice correlated with liver dysfunction, a detailed laboratory analysis was performed. Plasma samples of age-matched groups of the various $\mathrm{KO}$ and WT strains were analyzed for cholinesterase, alanine aminotransferase (ALT), aspartate aminotransfer- 


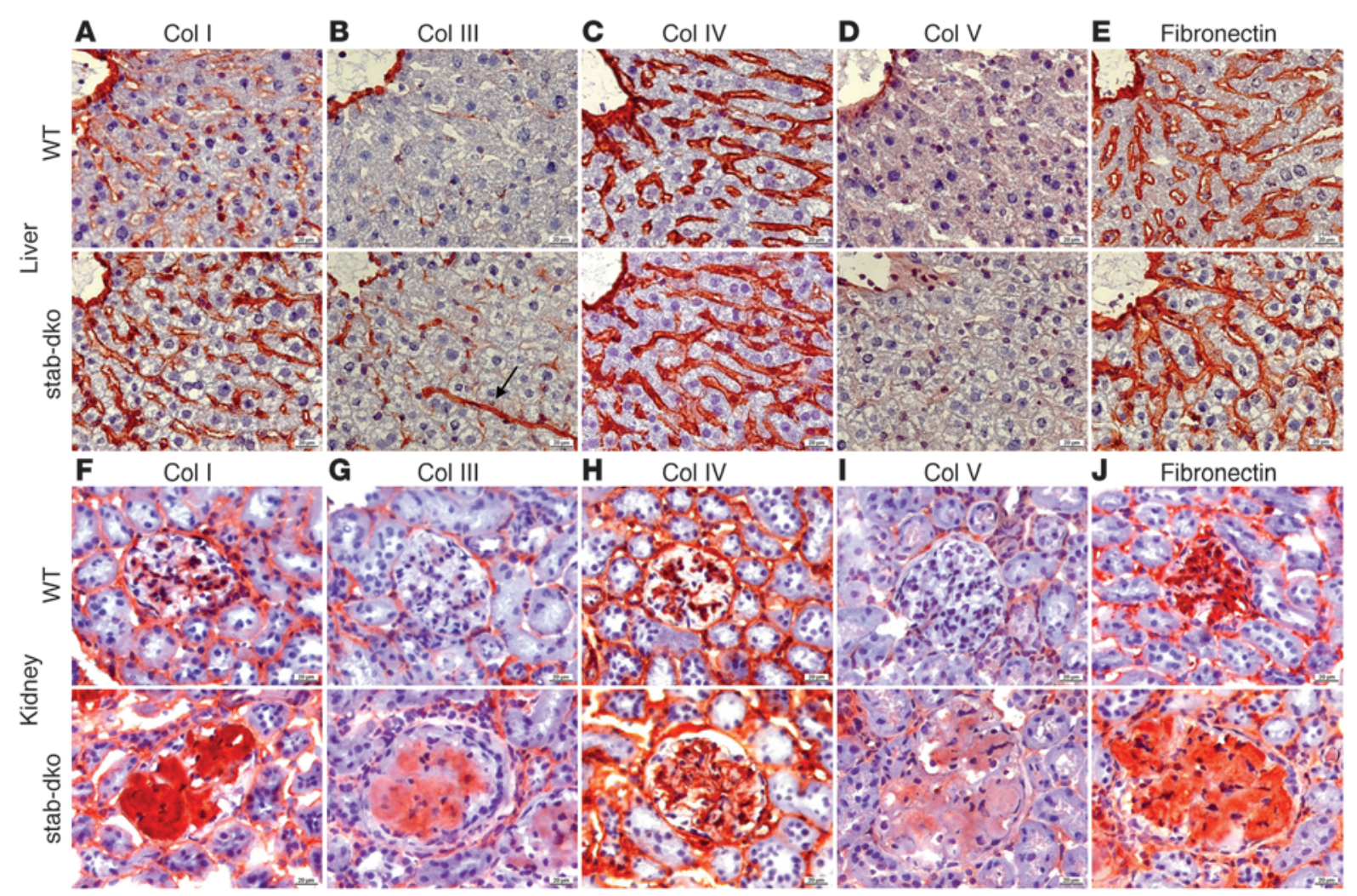

\section{Figure 4}

Analysis of extracellular matrix content in liver and kidney of stabilin-deficient mice. (A-E) Representative liver sections of 3-month-old C57BL/6 WT (first row) and C57BL/6 Stab1--Stab2-/- mice (second row) were stained by immunohistochemistry with antibodies against (A) collagen I, (B) collagen III, (C) collagen IV, (D) collagen V, and (E) fibronectin. Similar to the findings in Sirius red-stained samples, collagen III fibers (B, arrow) occurred along the sinusoidal network. (F-J) Representative kidney sections of 3-month-old C57BL/6 WT (third row) and C57BL/6 Stab1 $1^{-/}$Stab2 $2^{-/-}$mice (fourth row) were stained by immunohistochemistry with antibodies against (F) collagen I (G), collagen III (H), collagen IV $(\mathbf{I})$, collagen $\mathrm{V}$, and $(\mathbf{J})$ fibronectin. $(\mathbf{A}-\mathbf{J})$ The results of the immunohistochemical experiments are described in detail in Results. Original magnification, $\times 400$. Scale bars: $20 \mu \mathrm{m}$.

ase (AST), and glutamate dehydrogenase (GLDH) enzyme activity. While cholinesterase as a marker for liver synthetic function (Supplemental Table 2) and the liver transaminases (AST, ALT) as indicators for acute or chronic liver disease were always within normal range in all groups (Figure 2C), the plasma levels of GLDH increased in most, but not all, Stab1 $1^{-/-}$Stab2 $2^{-/-}$animals at 18 months of age (Figure 2D). GLDH is a mitochondrial enzyme that indicates tissue necrosis when detected in the peripheral blood; however, GLDH elevation is not specific for liver necrosis.

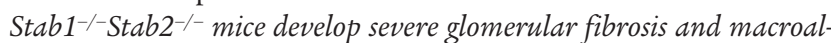
buminuria. As the observed perisinusoidal fibrotic liver phenotype in Stab1 $1^{-/-}$Stab2 $2^{-/-}$mice was special, but rather mild, a systematic survey for fibrotic and other histopathological changes was performed in kidney, heart, lung, brain, spleen, muscle, and bone using

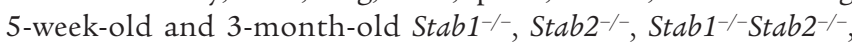
and WT animals. Except for the kidney, no major histopathologic abnormalities were detected at these early time points. Upon H\&E staining, the renal glomeruli in Stab1 $1^{-/}$Stab2 $2^{-/-}$mice, but not in Stab1 $1^{--}$, Stab2-/-, and WT mice increasingly enlarged over the 18 months of observation. Sirius red staining showed extensive collagen deposits and glomerular fibrosis in the kidneys of Stab1 $1^{-/}$Stab2 $2^{-1}$ mice, whereas Stab1 $1^{-/-}$and Stab2 $2^{-/-}$mice displayed a WT phenotype, even at 3 months of age (Figure 3B). PAS staining showed massive deposits of extracellular matrix and basement membrane material (Figure 3A), while amyloid was not detected by Kongo red staining (data not shown). Electron microscopy confirmed the development of large deposits of amorphous material and of collagen fibers in the mesangium of Stab1 $1^{-/-}$Stab2 $2^{-/-}$glomeruli (Figure 3C).

To determine whether kidney function was affected by the


kidney-related clinical chemistry parameters were investigated in plasma and urine: creatinine, albumin, urea, glucose, sodium, potassium, calcium, and phosphate. Most of the kidney values including plasma creatinine did not show significant differences in comparison to any of the KO strains or to WT controls (Supplemental Table 2). In the urine, already 3-and 6-month-old Stab1 $1^{-/-}$Stab2 $2^{-/-}$mice on Balb/c background showed highly elevated levels of albumin. While 3- and 6-month-old Stab1 ${ }^{-1-S t a b 2^{-}-}$ mice on C57BL/ 6 background showed only moderately elevated levels of albumin in the urine, very high albumin concentrations were measured in urine samples of 18-month-old Stab1-/-Stab2-/ animals (Figure 3D). In contrast, differences in plasma albumin concentration did not reach statistical significance (Supplemental Table 2). Thus, renal clearance function was not impaired by the glomerular fibrosis, but the progressive albuminuria suggested specific impairment of the glomerular filter function. 
A

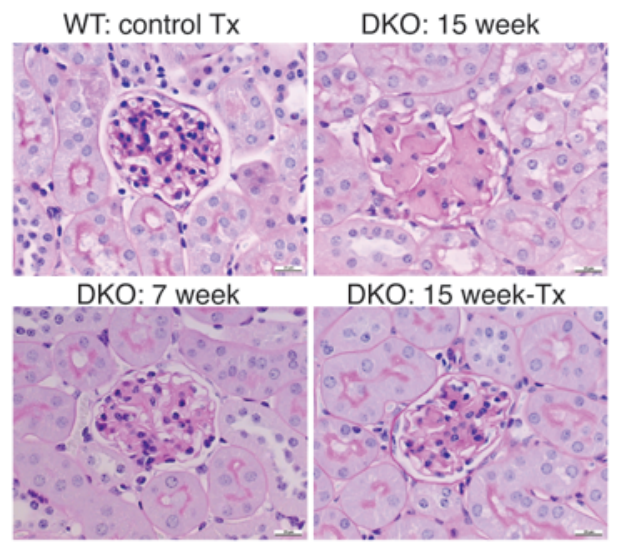

B

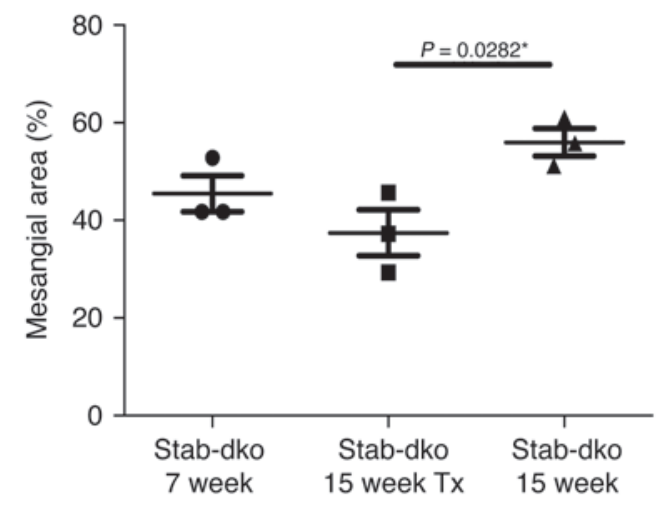

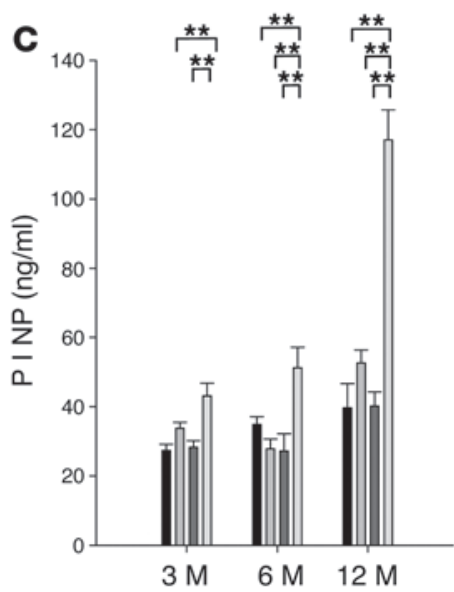
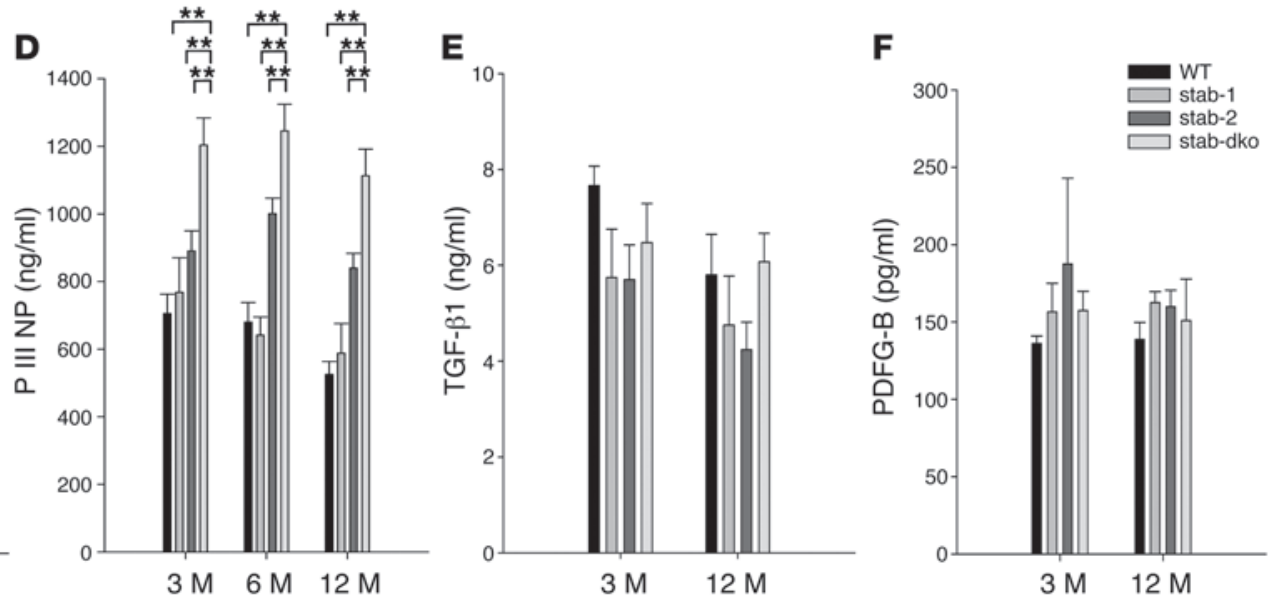

Figure 5

Effect of circulating factors on kidney phenotype. (A) Representative PAS-stained sections of kidneys from 7-week-old (lower left) and 15-week-old (upper right) Stab $1^{-1-S t a b 2} 2^{-/-}$mice and of Stab1 $1^{-1}$ Stab2 $2^{--}-$to-WT (lower right) as well as WT-to-WT (upper left) kidney transplants at 56 days after transplantation are shown $(n=3)$. While WT control transplants $($ Tx $)$ were unaffected and Stab1 $1^{-/}$Stab2 $2^{--}$glomeruli of 15 -week-old

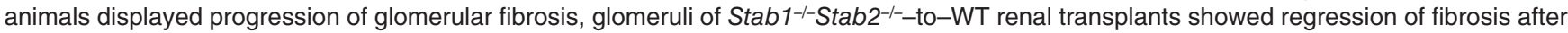
transplantation. Original magnification, $\times 400$. (B) Quantitative evaluation of glomerular fibrosis in Stab1-/-Stab2-/--to-WT renal transplants after transplantation $(n=3)$ showed a statistically significant decrease in mesangial area $(P=0.0282)$ as compared with 15 -week-old Stab1-/-Stab2 $2^{-/-}$ kidneys, while comparison with 7-week-old Stab1---Stab2-/- kidneys showed a tendency toward reduction of mesangial area. (C and D) Plasma samples of 3-, 6-, and 12-month-old WT, Stab1--, Stab2---, and Stab1--Stab2-/- mice of C57BL/6 genetic background were analyzed for P I NP and P III N) by ELISA. As only stabilin-2, but not stabilin-1, is a scavenger receptor for these propeptides, elevated levels of the propeptides

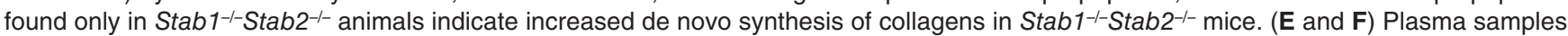

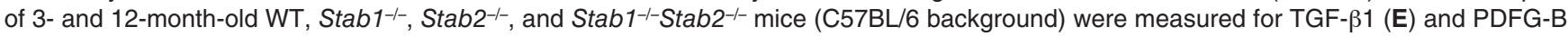
(F) by ELISA. No elevated levels of known profibrotic cytokines were detected in the tested stabilin animals Data represent mean \pm SEM for at least 5 mice per genotype. ${ }^{* *} P<0.01$.

Collagen III is expressed de novo in glomerular fibrosis and in perisinusoidal liver fibrosis in Stab1-/-Stab2-/- mice. In order to characterize the composition of the extracellular matrix deposited in perisinusoidal liver fibrosis and glomerular fibrosis in Stab1 $1^{-/}$Stab2 $2^{-/}$mice, an immunochemical analysis was performed on liver and kidney specimens using antibodies against collagen I, III, IV, V, and fibronectin. Stab1 $1^{-/}$and Stab2 $2^{--}$mice did not show marked differences in extracellular matrix composition when compared with WT animals (not shown). In contrast, collagen III (Figure 4B) was expressed de novo in Stab1 $1^{-/-}$Stab2 $2^{-/-}$livers forming similar perisinusoidal fibers as already detected by Sirius red staining; collagen I (Figure 4A) and fibronectin (Figure 4E) expressed at basal levels in WT liver sinusoids showed a moderate increase in expression, but did not colocalize with the collagen $\mathrm{III}^{+}$, Sirius red ${ }^{+}$perisinusoidal fibers. Collagen IV (Figure 4C) and collagen V (Figure 4D) appeared to

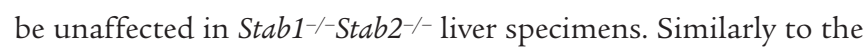
liver, de novo expression of collagen III (Figure 4G) as well as of collagen V (Figure 4I) was detected in the enlarged and fibrotic glomeruli of Stab1-/-Stab2-/- kidneys while collagen I was strongly increased (Figure 4F). Fibronectin expression (Figure 4J) was moderately increased in Stab1 $1^{-/}$Stab2 $2^{-/}$glomeruli, while no differences in expression were noticed for collagen IV (Figure 4H).

Progression to severe glomerular fibrosis is prevented by transplantation of kidneys from Stab1-/-Stab2-/- mice into WT animals. As stabilin-2 is not expressed in the kidney at all, while stabilin-1 expression in the kidney is restricted to rare connective tissue macrophages (Supplemental Figure 3), we reasoned that circulating rather than local factors might be responsible for the development of renal fibrosis. Therefore, kidneys from 7-week-old Stab1 $1^{-/ \text {Stab2 }}$ - $^{-/}$mice were transplanted orthotopically into 7-week-old WT recipients. At 15 weeks of age, the 
A
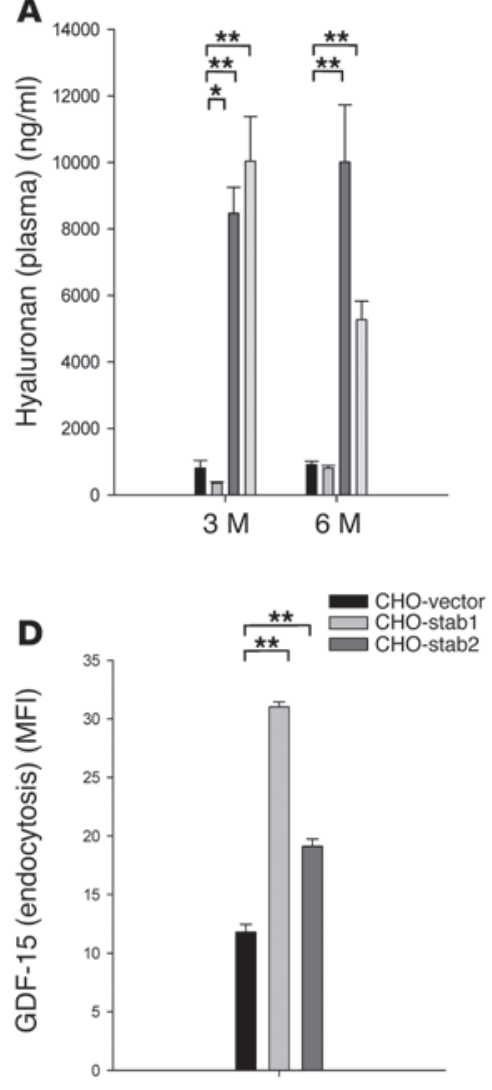

B
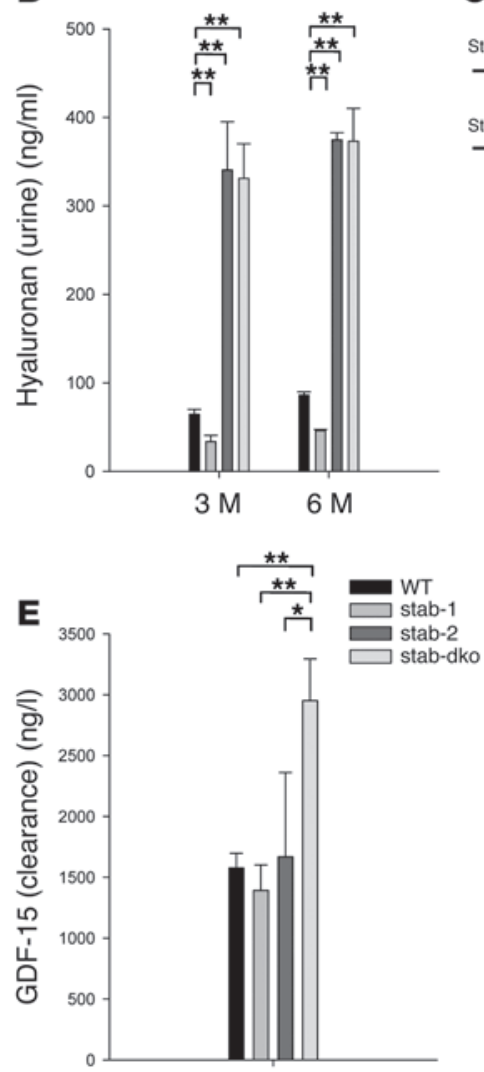

C

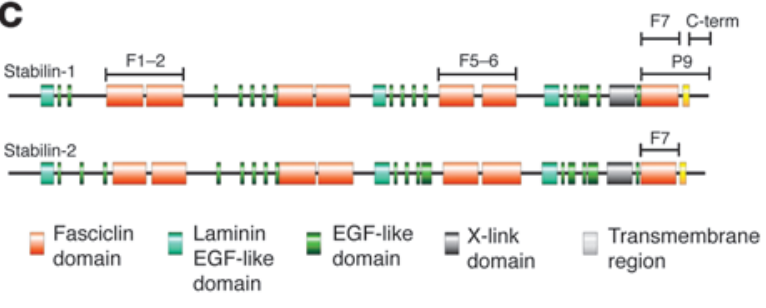

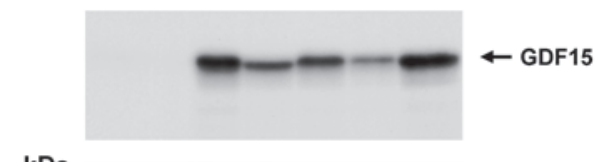

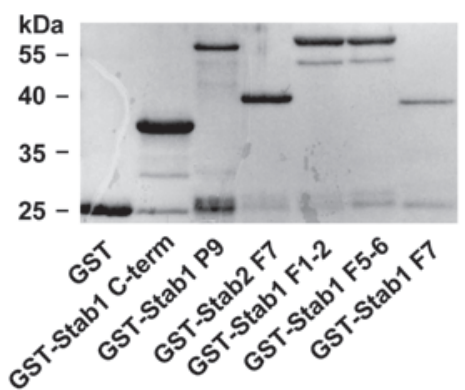

Figure 6

Differential impairment of hepatic blood clearance for stabilin ligands. (A and B) Plasma (A) and urine (B) HA levels of 3- and 6-month-old WT,

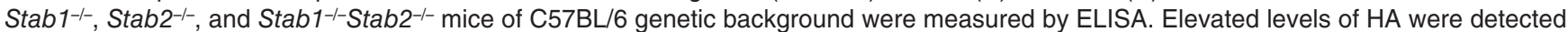

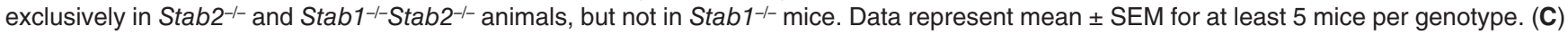
Schematic presentation of the protein domain organization of stabilin-1 and stabilin-2 shows the fragments used in the yeast 2-hybrid screening (P9) and GST-pull down assays. Stabilin-1 F1-2, stabilin-1 F5-6, stabilin-1 F7, stabilin-1 P9, stabilin-1 cytoplasmic tail (C-term), and stabilin-2 F7 are indicated by bars. GST pull-down assay demonstrating that fasciclin domains of both stabilin-1 and stabilin-2 interact with in vitro-translated GDF-15. GDF-15 neither binds to GST alone (negative control) nor to the GST-fused cytoplasmic tail of stabilin-1. $n=3$. (D) Quantification of stabilin-1- and stabilin-2-mediated uptake of Alexa Fluor 488-labeled GDF-15 using flow cytometry. $n=3$. Data represent mean \pm SEM for 3 independent experiments. (E) $5 \mu \mathrm{g}$ of recombinant human GDF-15 was injected in the tail vein of WT, Stab1 ${ }^{-1-}$, Stab2, and Stab1 $1^{-1-S t a b 2} 2^{-/-}$mice of Balb/c genetic background, and plasma levels of hGDF-15 were measured after 5 hours $(n=16, n=3, n=3, n=12$, respectively). Increased levels of hGDF-15 were exclusively observed in Stab1--Stab2-/- samples indicating impaired clearance of GDF-15 only upon combined absence of both stabilin receptors. Data represent mean \pm SEM. ${ }^{\star} P<0.05 ;{ }^{\star \star} P<0.01$.

recipients were sacrificed and the transplanted donor kidneys were compared with 7- and 15-week-old Stab1 $1^{-/}$Stab2 $2^{-1-}$ kidneys. Glomerular fibrosis in the transplanted donor kidneys 7 weeks after transplantation ( 15 weeks of age) showed a tendency toward improvement as compared with 7-week-old Stab1 $1^{-/-S t a b 2^{-/-}}$kidneys that did not reach statistical significance upon quantification of the mesangial area $(P=0.0867)$. In contrast, glomerular fibrosis in nontransplanted kidneys of 15 -week-old Stab1 $1^{-/}$Stab2 $2^{-/-}$mice showed severe progression of fibrosis as compared with the transplanted donor kidneys 56 days after transplantation (15 weeks of age) that was statistically significant $(P=0.0282)$ (Figure $5, \mathrm{~A}$ and $\mathrm{B})$. These findings indicate that glomerulofibrotic nephropathy is due to one or several circulat-

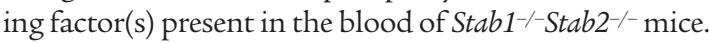

Biomarkers of tissue fibrosis, but not known profibrotic cytokines, are elevated in the circulation of Stab1 $1^{-1}$ Stab2 $2^{-/-}$mice. Biomarkers of fibrotic processes such as fibronectin and $\mathrm{N}$-terminal propeptides of collagen type I (P I NP) and type III (P III NP) correlated with glomeru- lar fibrosis. P I NP levels dramatically increased with age in Stab1-/Stab2 ${ }^{-/-}$mice, but not single-deficient or WT animals (Figure 5C). P III NP levels were stably elevated in 3-, 6-, and 12-month-old Stab1-/Stab2 ${ }^{-/-}$animals (Figure 5D), while fibronectin levels were slightly elevated (Supplemental Figure 4A). Altogether, increased plasma levels of biomarkers of fibrosis reflect the ongoing pathological processes in Stab1 $1^{-/-}$Stab2 $2^{-/-}$mice. As P I NP and P III NP are known ligands of stabilin-2, the increase in the circulation may also be due to decreased clearance of these molecules. Stab2 $2^{-1-}$ mice, however, did not show significantly increased P I NP or P III NP plasma lev-

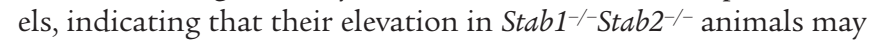
be mainly due to glomerular fibrosis. To address the question of whether development of fibrosis in Stab1 $1^{-/}$Stab2 $2^{-/-}$mice is related to any known profibrotic stimuli, we determined plasma levels of TGF- $\beta 1$, IGF, PDGF A, PDGF B, CTGF (not shown), IGFBP-2, and SPARC in 3- and 12-month-old animals (Figure 5, E and F, and Supplemental Figure 4, B-E). Whereas no differences were observed in 
plasma levels of TGF- $\beta 1$, PDGF-A and -B, CTGF and SPARC, minor changes were observed for IGF-1 and IGFBP2; however, these changes did not correlate with development of glomerular fibrosis.

Impaired hepatic blood clearance of HA in Stab2 $2^{-/}$, but not Stab1 $1^{-/}$mice. As Stabilin-2 is the dominant HA scavenger receptor of LSEC and as reduced clearance of HA might contribute to the development of the fibrotic tissue alterations observed in liver and kidney in

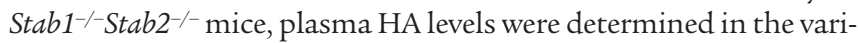
ous KO and WT strains. In both Stab2 $2^{-/-}$and Stab1 $1^{-/-}$Stab2 $2^{-/-}$mouse plasma, HA levels were increased by more than 10-fold (Figure 6A) as compared with Stab1-/- and WT mice (in case of 3-month-old WT, $810 \pm 227 \mathrm{ng} / \mathrm{ml}, n=5 ;$ Stab1 $^{-/-}, 361 \pm 81 \mathrm{ng} / \mathrm{ml}, n=5 ;$ Stab2 $2^{-/-}$, $8473 \pm 1743 \mathrm{ng} / \mathrm{ml}, n=5 ;$ Stab1 $1^{-/}$Stab2 $\left.{ }^{-/-}, 10039 \pm 360 \mathrm{ng} / \mathrm{ml}, n=5\right)$. In parallel, urine HA concentrations were also elevated in Stab2-/-

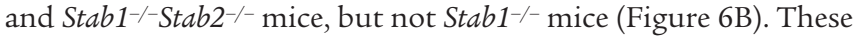
results clearly confirm the nonredundant function of stabilin-2 as the major HA clearance receptor of the body; as expected from molecular and biochemical data, stabilin-1 did not mediate HA clearance from the circulation under physiological conditions in vivo. These data further show that development of tissue fibrosis


HA levels associated solely with stabilin-2 deficiency.

Impaired hepatic blood clearance of GDF-15 in Stab1-/-Stab2-/- mice. In order to identify common endocytic ligands of both stabilin-1 and stabilin-2 whose impaired clearance might be linked to the pathology observed in Stab1 $1^{-/}$Stab2 $2^{-/}$mice, yeast 2-hybrid screening was used. The stabilin-1 fragment P9 was used as a bait; it did not activate transcription and was expressed at a high level in yeast (20). The P9 fragment contained 1 epidermal growth factor-like domain, the final fasciclin domain (F7), the transmembrane region, and the cytoplasmic tail of stabilin-1 (Figure 6). Screening of a human placental cDNA library under conditions of highest stringency resulted in identification of GDF-15 as an interacting partner of stabilin-1. GST pull-down assays confirmed the direct interaction between GDF-15 and the P9 fragment of stabilin-1 and demonstrated that this interaction is mediated by the fasciclin domain 7 (F7), but not by the cytoplasmic tail of stabilin-1 (domain overview shown in Figure 6C). Further testing of stabilin-1 fasciclin domains demonstrated that the interaction was not restricted to F7, indicating that it may be a general feature of the fasciclin domains of stabilin-1 to bind GDF-15. Due to the structural homology of the fasciclin domains, we reasoned that GDF-15 might also bind to the fasciclin domains of stabilin-2. Since the strongest binding of GDF-15 was observed with the F7 domain of stabilin-1, we selected the same domain of stabilin-2 for the GST pull-down assays and indeed demonstrated that stabilin-2-F7 interacts with GDF-15 (Figure 6C). In order to determine whether GDF-15 is a common endocytic ligand for both stabilins, we used a functional endocytosis assay that had been established by us previously $(17,21)$. $\mathrm{CHO}$ cells stably transfected with expression constructs for stabilin-1, stabilin-2, and empty vector were exposed for 30 minutes to Alexa Fluor 488-labeled GDF-15 at a final concentration of $10 \mu \mathrm{g} / \mathrm{ml}$. Flow cytometry quantification revealed a significantly increased uptake of Alexa Fluor 488-labeled GDF-15 by both stabilin-1- and stabilin-2-expressing cells (Figure 6D). Confocal microscopy analysis confirmed that the internalized ligand is transported into the endocytic pathway by both stabilins (Supplemental Figure 5). As these data indicated that GDF-15 is a common endocytic ligand for both stabilin-1 and stabilin-2, the question arose whether the clearance of GDF-15 is impaired in vivo in the absence of both stabilins.
Unfortunately, murine GDF-15 cannot be measured by ELISA due to lack of appropriate anti-murine GDF-15 antibodies. Instead, the clearance of recombinant human GDF-15 from the circulation was analyzed after injection into the tail vein of Stab1 $1^{-/-}$Stab2 $2^{-/-}$mice as well as of Stab1 $1^{-/-}$and Stab2 $2^{-/-}$mice and WT control animals; these experiments showed that the clearance of injected GDF-15 was significantly impaired only in Stab1 $1^{-/-}$Stab2 $2^{-/-}$mice (Figure 6E).

\section{Discussion}

In this study, we show that the simultaneous deficiency of the stabilin family of scavenger receptors comprising stabilin- 1 and stabilin-2 results in multiple pathological effects that include mild perisinusoidal hepatic fibrosis, severe glomerulofibrotic nephropathy, and a significantly decreased life span. In more general terms, this is the first time, to our knowledge, that it has been demonstrated that the hepatic clearance function has in vivo relevance for the homeostasis of the whole organism and that an impaired hepatic clearance function can even lead to premature death.

Stabilin-1 and stabilin-2 constitute a unique family of fasciclin domain-containing scavenger receptors. Despite high structural similarity of their extracellular ligand-interacting domains, several in vitro studies indicate that stabilin- 1 and -2 significantly differ in the repertoire of their endocytic ligands. Stabilin-2, for example, but not stabilin-1, is able to bind and internalize HA, AGE-modified proteins, and collagen N-terminal propeptides (12). In line with these findings, significantly increased HA levels in the circulation of stabilin-2-deficient mice demonstrated that a biochemically identified stabilin-2 endocytic ligand was indeed cleared by the receptor in vivo. In parallel, the normal HA levels in Stab1 $1^{-/-}$mice corresponded well to the absence of any interaction of stabilin-1 with HA in all in vitro systems tested $(12,24)$ and confirmed that HA binding by stabilin-2 is a nonredundant function that cannot be compensated for in vivo by any other scavenger receptor.

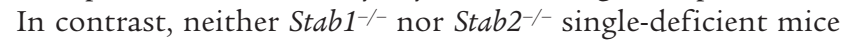
revealed any detectable pathological phenotype, suggesting that the decisive functions of the stabilins may be redundant, as has been suggested for other types of scavenger receptors $(25,26)$. In line with this notion, mice deficient for both stabilin-1 and stabilin-2 demonstrated an obvious and complex pathological phenotype. The life span of the double-deficient mice was significantly reduced. Both stabilin-1 and stabilin-2 are strongly coexpressed in LSECs that represent the major clearance machinery of the body for macromolecular waste molecules. Deficiency of both stabilins resulted in perisinusoidal hepatic fibrosis, posing the interesting question of whether locally produced or circulating factors or a combination of both result in local hepatic tissue injury when not correctly cleared by LSECs. With respect to this question, it was important to note that stabilin-1/2 double deficiency caused the most severe pathology in an organ distant to the liver, i.e., the kidney, showing severe glomerular fibrosis with massive mesangial deposition of collagens and of fibronectin that was accompanied by pronounced albuminuria. Expression of the stabilins in kidney is limited to single stabilin- $1^{+}$ macrophages, and there are no double stabilin- $1^{+} /$stabilin $-2^{+}$cells in this tissue. Thus, glomerular fibrosis may not be due to local deficiency in stabilin- $1 / 2$ function, but may rather be caused by (a) circulating factor or factors that evade clearance in the liver in Stab1 $1^{-/}$ Stab2 $2^{-1-}$ mice. This conclusion was further confirmed by kidney transplantation experiments showing that Stab1 $1^{-/}$Stab2 $2^{-/}$kidneys are prevented from further progression to severe glomerular fibrosis upon transplantation into WT recipients. 
In search for such a circulating profibrotic factor, several candidate molecules that have been reported to cause glomerulosclerosis have to be considered. TGF- $\beta 1$ has been described as inducing massive glomerulosclerosis and renal failure when overexpressed transgenically in hepatocytes accompanied by mild hepatic fibrosis and myocarditis resembling the findings in our double-deficient mice (27). TGF- $\beta 1$ acts by directly inducing mesangial matrix production and decreasing matrix remodeling as well as by inducing local TGF- $\beta$ overproduction (28). PDGF-B is another cytokine that may cause glomerulosclerosis, albeit rather by stimulating mesangial cell proliferation than matrix deposition (29). Beyond the direct actions of cytokines mediating glomerulosclerosis that besides TGF- $\beta 1$ and PDGF-B also comprise IGF-1, CTGF, and IGFBP2, extracellular matrix-derived factors may modulate induction and progression of glomerular fibrosis. The matricellular protein SPARC, for example, which has also been shown to be a ligand of stabilin-1 (17), is increased locally in the glomeruli during fibrotic processes and modulates TGF- $\beta 1$ and IGF- 1 activities (30). While TGF- $\beta 1$ and SPARC participate in a positive-feedback loop that is especially prominent in mesangial cells (31), SPARC rather downmodulates IGF-1 expression and signaling (32). For these reasons, these known profibrotic cytokines, including TGF- $\beta 1$, IGF-1, CTGF, PDGF-A and -B, and IGFBP2 as well as the matricellular protein SPARC, were tested in the plasma of Stab1 $1^{-/}$, Stab2 $2^{-/-}$ double-deficient mice; however, their levels remained unaltered as compared with WT animals.

As a consequence, we attempted to identify unknown common endocytic ligands of both stabilin-1 and -2 whose clearance is only impaired in double-deficient mice and could thus contribute to the pathologies seen in double- but not single-deficient mice. By using yeast 2-hybrid screening, in vitro protein-protein GST pull-down interaction assays and functional endocytosis assays in stably transfected CHO cells, a common endocytic ligand of stabilin-1 and -2, i.e., GDF-15, was identified. GDF-15, first described as macrophage inhibitory cytokine 1 (MIC-1) (33), is a distant member of the TGF- $\beta$ superfamily. Accumulating evidence indicates an association of GDF-15 with liver injury (34) and different malignancies including prostate, ovarian, and colorectal cancers $(35,36)$. More importantly, elevated plasma levels of GDF-15 are predictive of a high risk for mortality in human cardiovascular disease (37-39). Regarding the kidney, elevated levels of GDF-15 are associated with deterioration of kidney function as well as with overall and cardiovascular mortality in type 1 diabetic patients with nephropathy (40). In addition, there are several clues that GDF-15 may also be associated with fibrotic processes. In ethanol-induced liver injury, centrilobular expression of GDF-15 correlates with fibrosis and fatty change (34). In EP4-KO mice, increased levels of GDF-15 correlate with heart fibrosis and dilated cardiomyopathy (41). Thus, elevated levels of GDF-15 might parallel the pathological changes observed in Stab1 $1^{-/}$Stab2 $2^{-/}$mice. Since no reagents are available for the identification of mouse GDF-15 in the circulation, recombinant human GDF-15 was injected into the tail veins of Stab1 $1^{-/}$Stab2 $2^{-/}$mice, and the plasma levels of human GDF-15 were measured using a commercially available ELISA. Indeed, only double deficiency for both stabilins and not loss of either stabilin-1 or stabilin-2 alone resulted in impaired clearance of circulating human GDF-15. Nonetheless, GDF-15 is most likely not the decisive single factor causing glomerular damage in the double-deficient mice, as patients with thalassemia display more than 100-fold increased plasma levels of GDF-15 and do not show signs of renal damage (42). Identification and clearance of GDF-15 rather provide proof of principle for the further notion that there may be other ligands with a similar profile that are endocytosed by both stabilins (e.g., by binding to the homologous fasciclin domains) and that consequently only accumulate in the plasma of double-deficient mice. Identification of a single factor causing glomerulofibrotic nephropathy in Stab1 $1^{-/}$Stab2 $2^{-/}$mice may well be impossible, as only the interaction of several factors or their sequential actions might ultimately cause the disease.


resembles a rare human disease of unknown etiology, collagenofibrotic glomerulopathy (reviewed in ref. 43), characterized by massive glomerular deposition of type III collagen and proteinuria that may take a slowly progressive course toward end-stage renal failure in a subset of patients. Remarkably, matrix deposits are restricted to the glomeruli and may also contain collagen $\mathrm{V}$ and fibronectin that are produced by activated mesangial cells $(44,45)$; even more remarkably, collagenofibrotic glomerulopathy is viewed as a systemic disease with more widespread tissue fibrosis including the liver (46). The disease is extremely rare, and due to the fact that most patients described to date have been from Japan, a geographic, racial, or genetic preponderance may be assumed. In contrast to glomerulopathy with fibronectin deposits (47) or familial nephropathy with multiple exostoses (48), however, the pathological, molecular, and genetic mechanisms underlying collagenofibrotic nephropathy have not yet been elucidated.

Focal segmental glomerulosclerosis (FSGS), commonly associated with a nephrotic syndrome in humans, is histologically also characterized by glomerular deposition of collagen. Numerous etiologies have been identified for FSGS, such as familial/genetic, virus-associated, and drug-induced forms (49). In the so-called primary (idiopathic) FSGS, a yet-unidentified circulating permeability factor or factors are suspected. This hypothesis is based on several observations: (a) recurrence of FSGS after renal transplantation, (b) remission of proteinuria after plasmapheresis, and (c) sera of patients with FSGS being able to increase glomerular permeability in vitro and proteinuria in rats. Savin and colleagues were able to identify a FSGS permeability factor (FSPF) mostly consisting of low-molecular anionic glycoproteins, one of which may be cardiotrophin-like cytokine factor 1 (50-52). Nevertheless, unequivocal identification of a single factor as the decisive permeability-inducing and disease-causing factor in the FSPF has not yet been established. Therefore, it seems reasonable to assume that several factors interact or act in sequence to induce FSGS, some of which may even act indirectly to induce posttransplantation recurrence without directly influencing permeability, such as sST2 (53). In addition to the fact that the FSPF(s) are still elusive, the mechanisms of their accumulation in the circulation are so far unknown. As the clinical presentation and histologic alterations in FSGS bear some similarities to the pathology observed in Stab1 $1^{-/-}$ Stab2 $2^{--}$animals, we hypothesize that a similar mechanism may be operative. Decreased clearance of multiple factors may lead to accumulation of several ligands in the plasma and one or - more likely - several factors in combination may cause glomerular damage resulting in fibrosis. Thus, reduced hepatic blood clearance in Stab1 $1^{-/-}$Stab2 $2^{-/-}$mice offers a potential mechanism for the accumulation of FSPF(s) in primary/idiopathic FSGS.

In summary, we demonstrate here for what we believe is the first time that the stabilin family of scavenger receptors exert important nonredundant physiological functions in vivo. The deficien- 
cy of both stabilins results in multiple pathologies that may be caused by a circulating factor or factors that evade clearance by LSECs so that both stabilins contribute to tissue homeostasis not only in the liver, but also in other organs. GDF-15, a TGF- $\beta$ superfamily member, was identified here as a model ligand for such a circulating factor or factors, as it is a common ligand for both stabilins and its clearance is impaired only in the absence of the complete stabilin scavenger receptor system. The circulating factor or factors, however, that singly or in combination may cause

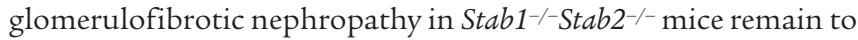
be identified. In conclusion, stabilin-1 and -2 together seem to guarantee proper clearance of the peripheral blood from potentially noxious agents and thus contribute to tissue homeostasis throughout the whole body.

\section{Methods}

Generation of Stab1 1/-, Stab2-/- mice. The stabilin-1 and stabilin-2 genes located on mouse chromosomes 14 and 10 are both organized in 69 exons. To generate mice deficient in stabilin- 1 and stabilin-2, we decided to target the minimal promoter region as well as the first exon, which contains the start codon. The targeting vectors (Supplemental Figure $1 \mathrm{~A}$ and Supplemental Figure $2 \mathrm{~A}$ ) were based on approximately $12-\mathrm{kb}$ genomic fragments flanking the promoter regions of both genes. In the case of stabilin-1, the targeting vector was constructed by placing a loxP site $0.6 \mathrm{~kb}$ upstream and a PGK neomycin phosphotransferase (NEO) selection cassette flanked by a loxP sequence $0.4 \mathrm{~kb}$ downstream of the first exon (Supplemental Figure 1A). In the case of stabilin-2, a loxP site flanked by a NEO selection cassette was introduced $1.3 \mathrm{~kb}$ upstream and a single loxP sequence was introduced $0.5 \mathrm{~kb}$ downstream of the start codon. After transfection of mouse embryonic stem cells (strain 129 S2), several single clones showing homologous recombination of the targeting vector into the appropriate stabilin allele were selected. After generation of chimeric and later on of stabilin-1 and - 2 mutant mice, the exon- 1 sequences flanked by loxP sites were removed by crossing the stabilin mutants with a cre-deleter mouse strain. Although the loxP sequences within the targeting vector would allow conditional strategies, all mice described in this study were fully deleted (promoter/

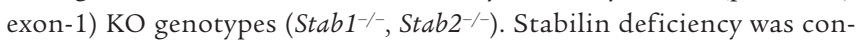
firmed on the DNA level by Southern blot analysis (see Supplemental Figure 1B and Supplemental Figure 2B) and on the protein level by immunohistochemistry (Figure 1A).

As primary mouse strains were generated in a mixed 129 and C57BL/6 genomic background, Stab $1^{-/-}$and Stab2 $2^{-/-}$mice were repetitively backcrossed to C57BL/6 and Balb/c inbred animals for at least 10 generations. All animal experiments including gene manipulations were approved by the Medical Ethics Committee II of the Medical Faculty Mannheim of the University of Heidelberg and filed with local authorities (Regierungspräsidium Karlsruhe).

Hङ E bistology and immunohistochemistry. Tissue samples were either fixed in formalin or shock frozen in OCT compound. For H\&E, PAS, silver, Kongo red, and Sirius red staining, formalin-fixed, paraffin-embedded samples were processed according to standard protocols. Frozen sections $(7 \mu \mathrm{m})$ were prepared as described (54), fixed 5 minutes in $-20^{\circ} \mathrm{C}$ acetone, and air dried. For immunohistochemical staining, tissue sections were rehydrated in PBS, $0.1 \%$ Tween 20, blocked in PBS, $1 \%$ BSA (Fitzgerald), and incubated for 1 hour with rabbit polyclonal antibodies against stabilin-1 (RS1; ref. 16), stabilin-2 (54), collagen I (Acris), collagen III (Acris), collagen IV (Genetex), collagen V (Abcam), or fibronectin (Abcam). After incubation with goat anti-rabbit-HRP (Santa Cruz Biotechnology Inc.), positive staining was visualized by AEC Envision Plus
(Dako). The slides were mounted with mounting medium (Dako) and photographed using a Leica microscope equipped with a DM500 digital camera and Openlab-3 software.

Electron microscopy. For liver specimens, the animals were perfused via the aorta and fixed with a cocktail of Ringers solution, $4 \%$ dextran, and $3 \%$ glutaraldehyde, as described previously (55). After fixation, the livers were removed and cut into sections of about $0.5 \mathrm{~mm}$ thickness with a razor blade. Appropriate specimens of altered liver tissue were selected under the stereomicroscope. From every liver, at least 10 specimens of 2 by $2 \mathrm{~mm}$ were cut with a razor blade, postfixed with $\mathrm{OsO} 4$, and embedded in Epon 812. Ultrathin sections were stained with uranyl acetate and lead citrate and were examined with a Phillips EM10 electron microscope. For kidney specimens, $1-\mathrm{mm}^{3}$ blocks of renal tissue were incubated 48 hours in Kanovsky fixative (2.0\% formaldehyde $2.5 \%$ glutaraldehyde buffered at $\mathrm{pH} 7.4$ with $0.2 \mathrm{~mol} / 1$ cacodylate). Samples were embedded in Araldite, and ultrathin sections were contrasted with lead citrate and viewed in an EM 912 (Zeiss).

Determination of blood and urine parameters. Animals of the same genetic background (Balb/c and C57BL/6) and of the same genotype (Stab1/-, Stab2 $2^{-/}$, Stab1 $1^{-/}$Stab2 $2^{-/}$and WT control mice) were arranged in groups of 6 to 8 and followed for an observation period of up to 2 years. At the age of 3 months, 6 months, 12 months and, if possible, at 18 months, mice were housed in metabolic cages for 24 hours to collect urine samples. The following parameters were analyzed routinely in urine samples in a Hitachi autoanalyzer: creatinine, urea, and glucose. Sodium, calcium, potassium, and phosphate levels were calculated photometrically (FLM-3; Radiometer). Mouse plasma was collected at the indicated time points and analyzed for the following routine parameters: creatinine, urea, cholinesterase, glucose, cholesterol, triglycerides, AST, ALT, GLDH, and gGT (Hitachi Autoanalyzer). Albumin levels in urine and plasma were determined by ELISA (see below). Protein concentrations were determined with the Bio-Rad protein assay kit (Bio-Rad) with BSA as a standard.

ELISA. Mouse TGF- $\beta 1$ (Hoelzel Biotech), IGFBP-2 (Mediagnost), PDGF-A (R\&D Systems), PDGF-B (R\&D Systems), IGF1 (Mediagnost), SPARC, P I NP, P III NP (all Hoelzel Biotech), and fibronectin (AssayPro) levels in mouse plasma as well as HA (R\&D Systems) levels in plasma and urine were measured according to the manufacturer's instructions. The albumin concentration in urine and plasma was determined as described previously (56) by use of a competitive 2-step ELISA including chicken anti-rat albumin antibody (Cappel) and a peroxidase-coupled antibody directed against chicken IgG (Sigma-Aldrich).

Yeast 2-bybrid screening. The yeast 2-hybrid screen was performed using the Matchmaker GAL4 Two-Hybrid system (BD Biosciences - Clontech). For bait generation, a fragment of stabilin-1 cDNA corresponding to aa 2302-2570 was amplified by PCR and cloned into the PDNR2 vector followed by subcloning into PLP-GBKT7 using the BD Creator cloning kit (BD Biosciences - Clontech). The bait protein was characterized according to the manufacturer's instructions; it was expressed on a high level, did not activate transcription in yeast, and did not affect the mating efficiency. The pretransformed human placenta Matchmaker cDNA library in the pGADT7-Rec vector, the Yeastmaker Yeast Transformation kit, and plasmid isolation kits were purchased from BD Biosciences - Clontech. Selection conditions of the highest stringency were used.

Endocytosis assay. Endocytosis of fluorescently labeled ligands in $\mathrm{CHO}$ cells stably expressing human stabilin-1 and stabilin-2 was performed as previously described (21). Generation of CHO-stabilin-1 and CHO-vector control cells has been described (15). The CHO-stabilin-2 cell line was generated as described for the CHO-stabilin-1 cell line using the pLPIRESneo-stabilin-2 expression construct. Purified recombinant human GDF-15 was purchased from R\&D Systems and labeled with Alexa Fluor 488 using the Alexa Fluor 488 Protein Labeling Kit (Invitrogen) accord- 
ing to the protocol of the manufacturer. As a positive control for endocytic activity, acLDL-Alexa Fluor 488 (Invitrogen) was used (data not shown). GDF-15-Alexa Fluor 488 was used at a concentration of $10 \mu \mathrm{g} / \mathrm{ml}$ for the endocytosis assay $\left(30\right.$ minutes at $\left.37^{\circ} \mathrm{C}\right)$. Cessation of endocytosis was achieved by placing the cells on ice or by immediate fixation in paraformaldehyde as described (19). For FACS analysis, CHO cells were incubated in culture dishes on ice for 15 minutes and gently recovered in ice-cold PBS. For immunofluorescent analysis, $\mathrm{CHO}$ cells were quickly washed 3 times with PBS without $\mathrm{Ca}^{2+}$ and $\mathrm{Mg}^{2+}$ and then subjected to fixation in paraformaldehyde.

FACS analysis. Quantification of bound/internalized fluorescent ligands was performed with FACSCalibur (BD Biosciences) according to standard protocols. Data were visualized and analyzed with WinMDI 2.8 software.

Immunofluorescence and confocal microscopy. The following primary antibodies were used: rabbit polyclonal anti-stabilin-1 RS1 (16) and mouse monoclonal anti-stabilin-2 clone 3.1 antibody (57). The corresponding secondary antibodies, conjugated with $\mathrm{Cy} 2, \mathrm{Cy} 3$, and $\mathrm{Cy} 5$, were from Dianova. Confocal microscopy was performed using the Leica TCS SP2 laser scanning spectral confocal microscope, equipped with a $63 \times 1.32$ objective. Excitation was with an argon laser emitting at $488 \mathrm{~nm}$, a krypton laser emitting at $568 \mathrm{~nm}$, and a helium/neon laser emitting at $633 \mathrm{~nm}$. Data were acquired and analyzed with Leica Confocal software. All 2- and 3 -color images were acquired by the use of a sequential scan mode.

In vitro protein-protein interactions. GST pull-down assays were performed as described (19). Fragments of stabilin- 1 corresponding to amino acids 2302 to 2570 (Stab1-P9), 2327 to 2463 (Stab1-F7), 385 to 644 (Stab1-F1-F2), and 1606 to 1867 (Stab1-F5-F6) and of stabilin-2 corresponding to amino acids 2293 to 2459 (Stab2-F7) were cloned into the EcoRI/XhoI sites of the pGEX4T1 vector (Invitrogen). GST-fused proteins were expressed in E. coli strain BL21-CodonPlus-RIL (Stratagene) and purified under nondenaturing conditions. PcDNA3-GDF-15 was used as a template to generate ${ }^{35} \mathrm{~S}$-methionine-labeled proteins by in vitro translation according to the standard protocol (Promega).

GDF-15 clearance. A total of $5 \mu \mathrm{g}$ recombinant human GDF-15 (carrier free variant; R\&D Systems) in $200 \mu \mathrm{l}$ PBS was injected intravenously in agematched 12- to 15-week-old Balb/c WT and Balb/c Stab1-/-Stab2-/- mice. Li-Heparin plasma samples were collected at 5 hours after injection from the retrobulbar area. GDF-15 levels were determined with an immunoluminometric assay, as previously described (37).
Kidney transplantation. Kidneys from 7-week-old C57BL/6 Stab1-/-Stab2-/donor mice were transplanted into age-matched C57BL/6 WT mice as described $(58)(n=3)$. For isograft controls C57BL/6 donor kidneys were transplanted into $\mathrm{C} 57 \mathrm{BL} / 6$ recipient mice $(n=3)$. Briefly, the right kidney attached to a segment of the aorta and of the renal vein along with the ureter was removed en bloc. The donor aorta and inferior vena cava were then anastomosed end-to-side to the recipient abdominal aorta and inferior vena cava below the level of the native renal vessels, respectively. The native left kidney was removed before revascularization. Donor and recipient ureter were anastomosed end-to-end at the border of the renal pelvis. The native right kidney was immediately removed after grafting. Animals did not receive any immunosuppressive therapy. Grafts were explanted and processed on posttransplantation day 56. For histology, graft samples and control samples were fixed in buffered $4 \%$ formaldehyde and embedded in paraffin.

Statistics. SigmaPlot 11 software was used to perform either $t$ test or 1-way ANOVA. $P<0.05$ was considered statistically significant. Error bars show the SEM of each experiment.

\section{Acknowledgments}

This work was supported in part by Deutsche Forschungsgemeinschaft (DFG) grants SFB405, project B12 (to S. Goerdt and J. Kzhyshkowska); SFB-TR23, project B1 (to S. Goerdt); SFB-TR77, project C3 (to S. Goerdt); and International Research Training Group grant GRK 880/3 of the DFG and The Netherlands Organisation for Scientific Research (projects 2 and 3 to S. Goerdt, K. Schledzewski, and J. Kzhyshkowska). We thank G. Küblbeck, P. Prochazka, E. Seelinger, P. Schwarz, V. Skude, and E. Wühl for continuous technical assistance and S. Kaden and Z. Antoni for excellent support in electron microscopy.

Received for publication August 12, 2010, and accepted in revised form November 10, 2010.

Address correspondence to: Cyrill Géraud, Klinik für Dermatologie, Venerologie und Allergologie, Universitätsmedizin Mannheim, Universität Heidelberg, D-68135 Mannheim, Germany. Phone: 49.621.383.2280; Fax: 49.621.383.3815; E-mail: cyrill.geraud@umm.de.
1. Smedsrod B. Clearance function of scavenger endothelial cells. Comp Hepatol. 2004;3 suppl 1:S22.

2. Seternes T, Sorensen K, Smedsrod B. Scavenger endothelial cells of vertebrates: a nonperipheral leukocyte system for high-capacity elimination of waste macromolecules. Proc Natl Acad Sci U S A. 2002;99(11):7594-7597.

3. Malovic I, et al. The mannose receptor on murine liver sinusoidal endothelial cells is the main denatured collagen clearance receptor. Hepatology. 2007;45(6):1454-1461.

4. Elvevold K, Simon-Santamaria J, Hasvold H, McCourt P, Smedsrod B, Sorensen KK. Liver sinusoidal endothelial cells depend on mannose receptor-mediated recruitment of lysosomal enzymes for normal degradation capacity. Hepatology. 2008;48(6):2007-2015.

5. Muro H, Shirasawa H, Kosugi I, Nakamura S. Defect of Fc receptors and phenotypical changes in sinusoidal endothelial cells in human liver cirrhosis. Am J Pathol. 1993;143(1):105-120.

6. Mousavi SA, Sporstol M, Fladeby C, Kjeken R, Barois N, Berg T. Receptor-mediated endocytosis of immune complexes in rat liver sinusoidal endothelial cells is mediated by FcgammaRIIb2. Hepatology. 2007;46(3):871-884.
7. March S, Hui EE, Underhill GH, Khetani S, Bhatia SN. Microenvironmental regulation of the sinusoidal endothelial cell phenotype in vitro. Hepatology. 2009;50(3):920-928.

8. Politz O, et al. Stabilin-1 and - 2 constitute a novel family of fasciclin-like hyaluronan receptor homologues. Biochem J. 2002;362(pt 1):155-164.

9. McCourt PA, Smedsrod BH, Melkko J, Johansson S. Characterization of a hyaluronan receptor on rat sinusoidal liver endothelial cells and its functional relationship to scavenger receptors. Hepatology. 1999;30(5):1276-1286.

10. Goerdt S, Bhardwaj R, Sorg C. Inducible expression of MS-1 high-molecular-weight protein by endothelial cells of continuous origin and by dendritic cells/macrophages in vivo and in vitro. $A m J$ Pathol. 1993;142(5):1409-1422.

11. Goerdt S, Walsh LJ, Murphy GF, Pober JS. Identification of a novel high molecular weight protein preferentially expressed by sinusoidal endothelial cells in normal human tissues. $J$ Cell Biol. 1991;113(6):1425-1437.

12. Hansen B, et al. Stabilin-1 and stabilin-2 are both directed into the early endocytic pathway in hepatic sinusoidal endothelium via interactions with clathrin/AP-2, independent of ligand binding. Exp Cell
Res. 2005;303(1):160-173

13. Harris EN, Baggenstoss BA, Weigel PH. Rat and human HARE/stabilin-2 are clearance receptors for high- and low-molecular-weight heparins. Am J Physiol Gastrointest Liver Physiol. 2009; 296(6):G1191-G1199.

14. Oie CI, Olsen R, Smedsrod B, Hansen JB. Liver sinusoidal endothelial cells are the principal site for elimination of unfractionated heparin from the circulation. Am J Physiol Gastrointest Liver Physiol. 2008;294(2):G520-G528.

15. Kzhyshkowska J, Gratchev A, Brundiers H, Mamidi S, Krusell L, Goerdt S. Phosphatidylinositide 3kinase activity is required for stabilin-1-mediated endosomal transport of acLDL. Immunobiology. 2005;210(2-4):161-173.

16. Kzhyshkowska J, et al. Alternatively activated macrophages regulate extracellular levels of the hormone placental lactogen via receptormediated uptake and transcytosis. I Immunol. 2008;180(5):3028-3037.

17. Kzhyshkowska J, et al. Novel function of alternatively activated macrophages: stabilin-1-mediated clearance of SPARC. JImmunol. 2006;176(10):5825-5832.

18. Falkowska-Hansen B, Falkowski M, Metharom P, Krunic D, Goerdt S. Clathrin-coated vesicles 
form a unique net-like structure in liver sinusoidal endothelial cells by assembling along undisrupted microtubules. Exp Cell Res. 2007;313(9):1745-1757.

19. Kzhyshkowska J, et al. Stabilin-1 localizes to endosomes and the trans-Golgi network in human mac rophages and interacts with GGA adaptors. J Leukoc Biol. 2004;76(6):1151-1161.

20. Kzhyshkowska J, et al. Novel stabilin-1 interacting chitinase-like protein (SI-CLP) is up-regulated in alternatively activated macrophages and secreted via lysosomal pathway. Blood. 2006;107(8):3221-3228.

21. Zhang J, et al. A novel GGA-binding site is required for intracellular sorting mediated by stabilin-1. Mol Cell Biol. 2009;29(22):6097-6105.

22. Karikoski M, et al. Clever-1/Stabilin-1 regulates lymphocyte migration within lymphatics and leukocyte entrance to sites of inflammation. Eur J Immunol. 2009;39(12):3477-3487.

23. Mouta Carreira C, et al. LYVE-1 is not restricted to the lymph vessels: expression in normal liver blood sinusoids and down-regulation in human liver cancer and cirrhosis. Cancer Res. 2001;61(22):8079-8084.

24. Kzhyshkowska J, Gratchev A, Goerdt S. Stabilin-1, a homeostatic scavenger receptor with multiple functions. J Cell Mol Med. 2006;10(3):635-649.

25. Peiser L, Mukhopadhyay S, Gordon S. Scavenger receptors in innate immunity. Curr Opin Immunol. 2002;14(1):123-128

26. Ling W, Lougheed M, Suzuki H, Buchan A, Kodama T, Steinbrecher UP. Oxidized or acetylated low density lipoproteins are rapidly cleared by the liver in mice with disruption of the scavenger receptor class A type I/II gene. J Clin Invest. 1997;100(2):244-252.

27. Sanderson N, et al. Hepatic expression of mature transforming growth factor beta 1 in transgenic mice results in multiple tissue lesions. Proc Natl Acad Sci US A. 1995;92(7):2572-2576.

28. Mozes MM, Bottinger EP, Jacot TA, Kopp JB. Renal expression of fibrotic matrix proteins and of transforming growth factor-beta (TGF-beta) isoforms in TGF-beta transgenic mice. J Am Soc Nephrol. 1999; 10(2):271-280.

29. Isaka Y, Fujiwara Y, Ueda N, Kaneda Y, Kamada $\mathrm{T}$, Imai E. Glomerulosclerosis induced by in vivo transfection of transforming growth factor-beta or platelet-derived growth factor gene into the rat kidney. J Clin Invest. 1993;92(6):2597-2601.

30. Francki A, Bradshaw AD, Bassuk JA, Howe CC, Couser WG, Sage EH. SPARC regulates the expression of collagen type I and transforming growth factor-beta1 in mesangial cells. J Biol Chem. 1999;274(45):32145-32152

31. Francki A, et al. SPARC regulates TGF-beta1- dependent signaling in primary glomerular mesangial cells. J Cell Biochem. 2004;91(5):915-925.

32. Francki A, et al. SPARC regulates cell cycle progression in mesangial cells via its inhibition of IGF-dependent signaling. J Cell Biochem. 2003;88(4):802-811.

33. Bootcov MR, et al. MIC-1, a novel macrophage inhibitory cytokine, is a divergent member of the TGF-beta superfamily. Proc Natl Acad Sci U S A. 1997; 94(21):11514-11519.

34. Hsiao EC, Koniaris LG, Zimmers-Koniaris T, Sebald SM, Huynh TV, Lee SJ. Characterization of growthdifferentiation factor 15 , a transforming growth factor beta superfamily member induced following liver injury. Mol Cell Biol. 2000;20(10):3742-3751.

35. Xue $\mathrm{H}$, et al. Identification of serum biomarkers for colorectal cancer metastasis using a differential secretome approach. J Proteome Res. 2010;9(1):545-555.

36. Brown DA, et al. Macrophage inhibitory cytokine 1 : a new prognostic marker in prostate cancer. Clin Cancer Res. 2009;15(21):6658-6664.

37. KempfT, et al. Circulating concentrations of growthdifferentiation factor 15 in apparently healthy elderly individuals and patients with chronic heart failure as assessed by a new immunoradiometric sandwich assay. Clin Chem. 2007;53(2):284-291.

38. Kempf T, et al. Growth-differentiation factor-15 for risk stratification in patients with stable and unstable coronary heart disease: results from the AtheroGene study. Circ Cardiovasc Genet. 2009;2(3):286-292.

39. Wollert KC, et al. Growth differentiation factor 15 for risk stratification and selection of an invasive treatment strategy in non ST-elevation acute coronary syndrome. Circulation. 2007;116(14):1540-1548.

40. Lajer M, Jorsal A, Tarnow L, Parving HH, Rossing P. Plasma growth differentiation factor- 15 independently predicts all-cause and cardiovascular mortality as well as deterioration of kidney function in type 1 diabetic patients with nephropathy. Diabetes Care. 2010;33(7):1567-1572.

41. Harding P, Yang XP, Yang J, Shesely E, He Q, LaPointe MC. Gene expression profiling of dilated cardiomyopathy in older male EP4 knockout mice. Am JPhysiol Heart Circ Physiol. 2010;298(2):H623-H632.

42. Tanno T, et al. High levels of GDF15 in thalassemia suppress expression of the iron regulatory protein hepcidin. Nat Med. 2007;13(9):1096-1101.

43. Alchi B, Nishi S, Narita I, Gejyo F. Collagenofibrotic glomerulopathy: clinicopathologic overview of a rare glomerular disease. Am J Kidney Dis. 2007:49(4):499-506.

44. Naruse K, et al. Mesangial cell activation in the collagenofibrotic glomerulonephropathy. Case report and review of the literature. Virchows Arch.
1998;433(2):183-188

45. Morita $\mathrm{H}$, et al. Collagenofibrotic glomerulopathy with a widespread expression of type-V collagen. Virchows Arch. 2003;442(2):163-168.

46. Yasuda T, et al. Collagenofibrotic glomerulopathy: a systemic disease. Am J Kidney Dis. 1999;33(1):123-127.

47. Castelletti F, et al. Mutations in FN1 cause glomerulopathy with fibronectin deposits. Proc Natl Acad Sci U S A. 2008;105(7):2538-2543.

48. Roberts IS, Gleadle JM. Familial nephropathy and multiple exostoses with exostosin-1 (EXT1) gene mutation. J Am Soc Nephrol. 2008;19(3):450-453.

49. D'Agati VD. The spectrum of focal segmental glomerulosclerosis: new insights. Curr Opin Nephrol Hypertens. 2008;17(3):271-281.

50. Sharma M, Sharma R, McCarthy ET, Savin VJ. "The FSGS factor:" enrichment and in vivo effect of activity from focal segmental glomerulosclerosis plasma. J Am Soc Nephrol. 1999;10(3):552-561.

51. Savin VJ, McCarthy ET, Sharma M. Permeability factors in focal segmental glomerulosclerosis. Semin Nephrol. 2003;23(2):147-160.

52. Savin VJ, Sharma M. Plasma "factors" in recurrent nephrotic syndrome after kidney transplantation: causes or consequences of glomerular injury? Am J Kidney Dis. 2009;54(3):406-409.

53 . Bruneau S, et al. Potential role of soluble ST2 protein in idiopathic nephrotic syndrome recurrence following kidney transplantation. Am J Kidney Dis. 2009;54(3):522-532.

54. Falkowski M, Schledzewski K, Hansen B, Goerdt S. Expression of stabilin-2, a novel fasciclinlike hyaluronan receptor protein, in murine sinusoidal endothelia, avascular tissues, and at solid/liquid interfaces. Histochem Cell Biol. 2003; 120(5):361-369.

55. Dombrowski F, Lehringer-Polzin M, Pfeifer U. Hyperproliferative liver acini after intraportal islet transplantation in streptozotocin-induced diabetic rats. Lab Invest. 1994;71(5):688-699.

56. Obermuller N, Kranzlin B, Blum WF, Gretz N, Witzgall R. An endocytosis defect as a possible cause of proteinuria in polycystic kidney disease. $A m J$ Physiol Renal Physiol. 2001;280(2):F244-F253.

57. Martens JH, et al. Differential expression of a gene signature for scavenger/lectin receptors by endothelial cells and macrophages in human lymph node sinuses, the primary sites of regional metastasis. J Pathol. 2006;208(4):574-589.

58. Wang S, et al. Recipient Toll-like receptors contribute to chronic graft dysfunction by both MyD88and TRIF-dependent signaling. Dis Model Mech. 2010;3(1-2):92-103. 